\title{
Reconfigurable network for quantum transport simulations
}

\author{
Mario A. Quiroz-Juárez $\odot,{ }^{1,2, *}$ Chenglong You $\odot,{ }^{3}$ Javier Carrillo-Martínez $\odot,{ }^{1}$ Diego Montiel-Álvarez, ${ }^{1}$ José L. Aragón, ${ }^{4}$ \\ Omar S. Magaña-Loaiza, ${ }^{3, \dagger}$ and Roberto de J. León-Montiel $\oplus^{1, *}$ \\ ${ }^{1}$ Instituto de Ciencias Nucleares, Universidad Nacional Autónoma de México, Apartado Postal 70-543, 04510 Ciudad México, México \\ ${ }^{2}$ Departamento de Física, Universidad Autónoma Metropolitana Unidad Iztapalapa, San Rafael Atlixco 186, 09340 Ciudad México, México \\ ${ }^{3}$ Quantum Photonics Laboratory, Department of Physics and Astronomy, Louisiana State University, Baton Rouge, Louisiana 70803, USA \\ ${ }^{4}$ Centro de Física Aplicada y Tecnología Avanzada, Universidad Nacional Autónoma de México, Boulevard Juriquilla 3001,
} 76230 Querétaro, México

(Received 27 July 2020; revised 14 October 2020; accepted 1 December 2020; published 6 January 2021)

\begin{abstract}
We put forward a versatile, highly scalable, tunable electronic platform for the simulation of single-excitation quantum transport phenomena. Our system, comprising 10 state-of-the-art, fully reconfigurable electronic oscillators, is implemented by making use of functional blocks synthesized with operational amplifiers and passive linear electrical components. To test the robustness and precise control of our platform, we simulate different quantum transport protocols, such as the ballistic propagation of a single-excitation wave function in an ordered lattice, and its localization due to disorder. We implement the Su-Schrieffer-Heeger model to directly observe the emergence of topologically protected one-dimensional edge states. Furthermore, we present the realization of the so-called perfect transport protocol, a key milestone for the development of scalable quantum computing and communication. Finally, we show a simulation of the exciton dynamics in the B800 ring of the purple bacteria LH2 complex. The high fidelity of our simulations together with the low decoherence of our device make it a robust, versatile, and promising platform for the simulation of quantum transport phenomena.
\end{abstract}

DOI: 10.1103/PhysRevResearch.3.013010

\section{INTRODUCTION}

Understanding the limits of controllability of quantum and classical transport has long been considered a topic of great relevance in physics, chemistry, and biology [1-3]. In particular, the study of novel materials that exhibit excitation-energy transfer pathways, which are different from those available in nature, has recently attracted a great deal of attention. Indeed, the control of transport phenomena at the nanoscale has shown an enormous potential for the development of new light-harvesting technologies for solar energy conversion [4], enhanced sensing [5-7], and even for the design of electronic and photonic circuits capable of performing complex tasks with high efficiency [8-11]. In this regard, quantum (and classical) random walks have emerged as useful models for the experimental simulation of nontrivial transport phenomena in physical systems. Random walks have been implemented in different platforms, such as optical cavities [12-14], trapped ions [15-18], ultracold atomic lattices [19-22], superconducting circuits [23-25], optical tweezers [26-30], and integrated photonics [31-40]. The latter has been particularly attractive due to its low loss and high interferometric visibilities, as well

\footnotetext{
*mario.quiroz@correo.nucleares.unam.mx

†maganaloaiza@1su.edu

†roberto.leon@nucleares.unam.mx
}

Published by the American Physical Society under the terms of the Creative Commons Attribution 4.0 International license. Further distribution of this work must maintain attribution to the author(s) and the published article's title, journal citation, and DOI. as the possibility of scaling it to many active and reconfigurable components [41].

In this work, we demonstrate robust simulation of quantum transport using a state-of-the-art reconfigurable electronic network. This is managed by constructing a unique mapping that allows us to establish a direct connection between the probability amplitudes of a quantum tight-binding system and the voltages of coupled electrical-oscillator networks. Our platform, which comprises 10 fully reconfigurable $R L C$ oscillators, is implemented by means of operational amplifiers and passive linear electrical components. This let us operate, as many of the aforementioned platforms, within the singleexcitation Hilbert subspace, i.e., the space that describes the dynamics of a single particle in a tight-binding quantum network [42].

To test the versatility and precision of our platform, we have implemented different quantum transport protocols that demand specific site frequencies and coupling conditions. In particular, we have explored the ballistic propagation of a single-excitation wave function in an ordered lattice and its localization due to stochastically varying couplings (static disorder), i.e., the so-called Anderson localization [43]. We have implemented the Su-Schrieffer-Heeger (SSH) model [44,45], where the proper use of alternating-coupling values, and fixed site energies, allows us to directly observe the emergence of one-dimensional edge states. Because of its relevance for scalable quantum computing and communication [46,47], we have implemented the protocol known as perfect transport [48-51], which makes use of a linear chain of qubits (or sites) where the couplings between them follow a precise squareroot rule to coherently transfer quantum states. Furthermore, 
we have tested our platform capabilities for mimicking the transport behavior of photosynthetic light-harvesting complexes by implementing a simulation of the exciton dynamics in the B800 ring of the purple bacteria LH2 complex [52]. Finally, we show the capability of our platform to mimic twodimensional lattices comprising independently addressable long-range interactions. This is demonstrated by simulating the exciton dynamics in a modified B800 ring with artificially introduced couplings between far-lying sites.

\section{THEORETICAL MODEL}

The dynamics of a single excitation in a system comprising $N$ coupled quantum oscillators is described by the Schrodinger equation $i \partial_{t}|\psi(t)\rangle=\hat{H}|\psi(t)\rangle$, where the Hamiltonian is given by

$$
\hat{H}=\sum_{n=1}^{N} \varepsilon_{n}|n\rangle\left\langle n\left|+\sum_{n \neq m}^{N} J_{n m}\right| n\right\rangle\langle m|,
$$

with $|n\rangle$ denoting the energy density associated to the $n$th oscillator. The $n$ th-site energies and the coupling between sites $n$ and $m$ are given by $\varepsilon_{n}$ and $J_{n m}$, respectively. Then, by expanding the time-dependent wave function in the site basis, i.e., $|\psi(t)\rangle=\sum_{n} c_{n}(t)|n\rangle$, it is straightforward to find that the Schrodinger equation leads to a set of first-order coupled differential equations of the form $i \partial_{t} c_{n}=\varepsilon_{n} c_{n}+\sum_{n \neq m}^{N} J_{n m} c_{m}$. In the weak-coupling limit $\left(J_{n m} \ll \varepsilon_{n}\right)$, the time derivative of this equation becomes $[53,54]$

$$
\frac{d^{2} c_{n}}{d t^{2}}=-\varepsilon_{n}^{2} c_{n}-\varepsilon_{n} \sum_{n \neq m}^{N} 2 J_{n m} c_{m}
$$

As we will show below, the importance of this expression resides in the fact that it allows us to establish a direct connection between the probability amplitudes $c_{n}$ of a quantum system and the voltages $V_{n}$ in an electrical-oscillator network. To do so, let us consider an array of $N$ inductively coupled $R L C$ oscillators (see Appendix A for details), where $R, L$, and $C$ stand for resistor, inductor, and capacitor, respectively. We can use the Kirchhoff laws to find that the equations of motion for the voltages $V_{n}(t)$ across the capacitors $C_{n}$ are given by

$$
\begin{aligned}
\frac{d^{2} V_{n}}{d t^{2}}= & \frac{1}{C_{n}}\left(-\frac{1}{R_{n}} \frac{d V_{n}}{d t}-\frac{V_{n}}{L_{n}}-\sum_{j=n+1}^{N} \frac{V_{n}-V_{j}}{L_{n j}}\right. \\
& \left.+\sum_{j=1}^{j<n} \frac{V_{j}-V_{n}}{L_{j n}}\right),
\end{aligned}
$$

where $L_{n j}$ stands for the inductor that couples the $n$th and jth oscillators. Remarkably, by writing Eq. (3) in the nondissipative limit, i.e., when $R \rightarrow \infty$, one can find that it is mathematically equivalent to Eq. (2) with

$$
\varepsilon_{n}^{2}=\frac{1}{C_{n}}\left(\frac{1}{L_{n}}+\sum_{m \neq n}^{N} \frac{1}{L_{n m}}\right), \quad J_{n m}=-\frac{1}{2 \varepsilon_{n} L_{n m} C_{n}} .
$$

Note that this mapping among probability amplitudes $c_{n}(t)$ and voltages $V_{n}(t)$ allows for implementing one- and twodimensional lattices with couplings beyond the nearest- neighbor interaction. Interestingly, we can account for the parasitic losses that are present in its experimental implementation. This can be performed by adding a non-Hermitian term to the Hamiltonian (1). This non-Hermitian term is determined by analyzing the time-dependent energy in the quantum and electronic models. While the total energy in the quantum system is given by $Q_{\mathrm{q}}(t)=\sum_{n}\left|c_{n}\right|^{2}$, the energy stored in and across the 10 coupled oscillators is obtained by writing $Q_{\mathrm{cl}}(t)=\frac{1}{2} \sum_{m \neq n} C_{n} V_{n}^{2}+L_{n} I_{n}^{2}+L_{n m} I_{n m}^{2}$, where $I_{n}$ and $I_{n m}$ stand for the currents passing through the oscillator and coupling inductors, respectively. By tracking time traces for both energies, one can find that the energy decay rates keep a quantitative agreement if the term $\hat{H}_{\text {loss }}=-\frac{i}{2} \sum_{n} \Gamma_{n}|n\rangle\langle n|$, with $\Gamma_{n}=1 /\left(R_{n} C_{n}\right)$ describing the rate at which energy is dissipated, is included in the Hamiltonian (1); see Appendix B for details.

\section{EXPERIMENTAL RESULTS}

Our current version of the electronic platform comprises 10 fully reconfigurable $R L C$ oscillators, where site frequencies and couplings can be independently selected from a broad range of possible values (see Appendix $A$ and Refs. [55-57] for details). This allows us to explore different quantum transport protocols, including Anderson localization, the emergence of edge states in the SSH model, the coherent transfer of a quantum state, and the simulation of excitonic-energy transport in photosynthetic light-harvesting complexes.

Anderson localization. The localization of a particle's wave function in disordered lattices is one of the most fascinating effects in physics [43]. This fundamental phenomenon, known as Anderson localization, arises from the interference of multiple scattering effects. In this scenario, the wave function of a propagating particle in a lattice is affected by static disorder, introduced in either the lattice-site energies (diagonal disorder) or in the coupling among them (off-diagonal disorder) [58]. We have implemented the Anderson localization protocol by making use of $N=9$ out of the 10 available oscillators in our electronic platform. The oscillators are arranged in a one-dimensional nearest-neighbor-coupled lattice described by the Hamiltonian

$$
\begin{aligned}
\hat{H}_{\mathrm{AL}}= & \sum_{n=1}^{N}\left(\varepsilon_{n}-i \Gamma_{n}\right)|n\rangle\left\langle n\left|+\sum_{n=1}^{N-1} J_{n, n+1}\right| n\right\rangle\langle n+1| \\
& +\sum_{n=1}^{N-1} J_{n+1, n}|n+1\rangle\langle n| .
\end{aligned}
$$

All site frequencies $\varepsilon_{n}$ and losses $\Gamma_{n}$ are described by the values presented in the first row of Table I in Appendix A. The static disorder is introduced through the coupling between sites by randomly selecting the value of each coupling inductor from a uniform distribution. This is described by $\left[L_{x}(1-\Delta), L_{x}(1+\Delta)\right]$, with $L_{x}=96.05 \mathrm{mH}$ and $\Delta=$ $0,0.5,0.9$ indicating the degree of the lattice disorder. Figure 1 shows the time evolution of an excitation (voltage signal) initialized in the central site of the one-dimensional lattice. The first column shows the quantum mechanically predicted population $\left|c_{n}\right|^{2}$ evolution, whereas the second column 

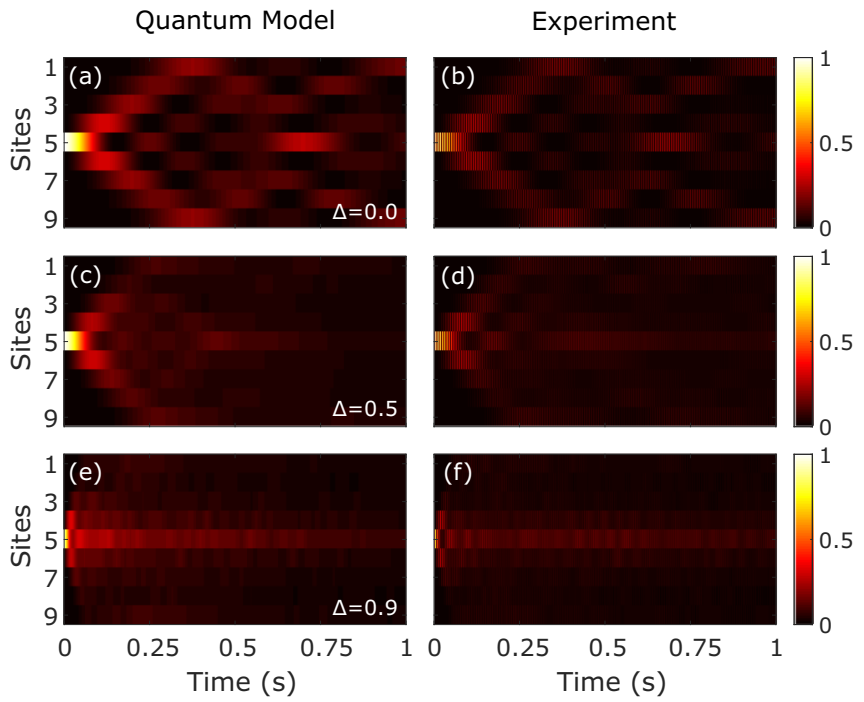

FIG. 1. Time evolution of an excitation (voltage signal) initialized in the central site of a one-dimensional network comprising nine nearest-neighbor-coupled oscillators. The rows (from top to bottom) depict the evolution for increasingly larger degree of disorder: (a), (b) $\Delta=0$, (c), (d) $\Delta=0.5$, and (e), (f) $\Delta=0.9$. The results presented in all panels correspond to the average of 50 different disorderedarray time evolutions.

shows our experimentally obtained squared-voltage-signal $\left|V_{n}\right|^{2}$ evolution. Notice that as one might expect, ballistic propagation of the excitation is observed when disorder is absent $(\Delta=0)$ [see Figs. 1(a) and 1(b)], while for strong disorder $(\Delta=0.9)$, the excitation gets localized in the central site of the lattice, as depicted in Figs. 1(e) and 1(f). It is important to remark that given the stochastic nature of Anderson localization, the results shown in each panel of Fig. 1 correspond to the average of 50 different disordered-array time evolutions.

The Su-Schrieffer-Heeger (SSH) model. One of the simplest models to study nontrivial topological phenomena, such as the emergence of topologically protected edge states, is the Su-Schrieffer-Heeger (SSH) model [44,45]. The SSH model describes the hopping of a spinless fermion on a one-dimensional lattice with staggered hopping amplitudes, as shown in the insets of Fig. 2. The chain consists of $N$ unit cells, each of which hosts two sites, i.e., one on sublattice $A$ and one on sublattice $B$. We neglect interactions between electrons; consequently, the dynamics of each electron is described by a single-excitation Hamiltonian of the form [59]

$$
\begin{aligned}
\hat{H}_{\mathrm{SSH}}= & \sum_{n=1}^{N}\left[\left(\varepsilon_{n}-i \Gamma_{n}\right)|n\rangle\langle n|+J_{\alpha}(|n, B\rangle\langle n, A|+\text { H.c. })\right] \\
& +J_{\beta} \sum_{n=1}^{N-1}(|n+1, A\rangle\langle n, B|+\text { H.c. }),
\end{aligned}
$$

where the states of the chain are described by $|n, A\rangle$ and $|n, B\rangle$, with the electron's unit cell represented by $n \in\{1,2, \ldots, N\}$, and H.c. stands for the Hermitian conjugate.

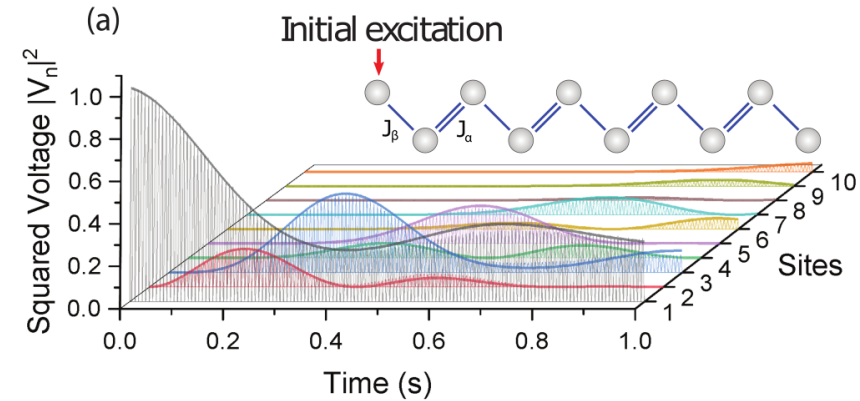

(b) Initial excitation

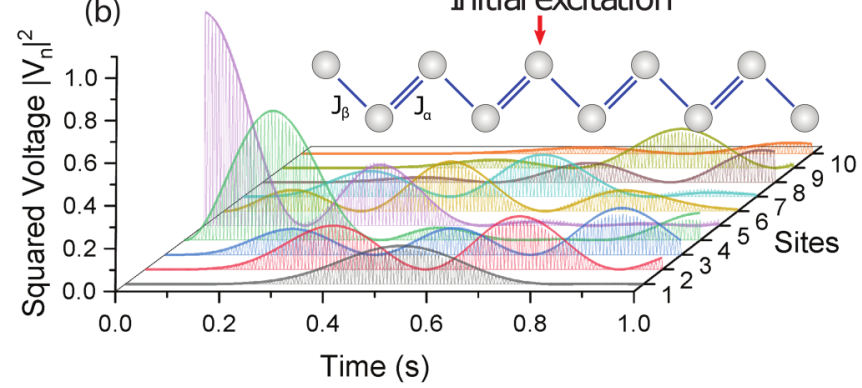

(c) Initial excitation

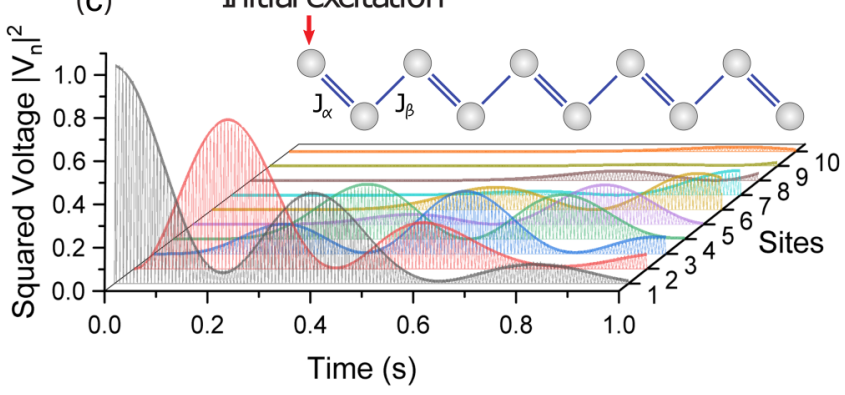

FIG. 2. Time evolution of a voltage signal (excitation) initialized in (a) the edge and (b) the bulk of a 10-oscillator Su-SchriefferHeeger (SSH) chain. (c) The dynamics of an excitation initialized at the edge of the system in the trivial phase, i.e., with the opposite bond dimerization pattern. The fast oscillating signals correspond to the experimentally measured squared voltages $\left|V_{n}\right|^{2}$, whereas the slowly varying envelope (solid lines) shows the theoretically predicted behavior of the quantum populations $\left|c_{n}\right|^{2}$. The insets show the lattice structure, as well as the initial excitation conditions in each case.

Arguably, the most important feature of the SSH model is the emergence of topologically protected edge modes at the end of the chain, when the intracell coupling $J_{\alpha}$ exceeds the intercell coupling $J_{\beta}$ [60]. We have experimentally produced these states by making use of all 10 sites in our electronic platform. The parameters used for implementing the Hamiltonian in Eq. (6) are described in the second row of Table I in Appendix A. Note that the coupling inductors satisfy the condition $J_{\alpha}=2 J_{\beta}$. Figure 2 shows the time evolution of an excitation (voltage signal) initialized in the edge [Fig. 2(a)] and the bulk [Fig. 2(b)] of the chain. Note that the fast oscillating signals correspond to the experimentally measured squared voltages $\left|V_{n}\right|^{2}$. The slowly varying envelope (solid lines) shows the theoretically predicted behavior of the quantum populations $\left|c_{n}\right|^{2}$. These results demonstrate two important facts: (1) the quantum probabilities follow the same dynamics as the envelope of the squared voltage signals, and (2) the small frequencies used in our device $(\sim 1.5 \mathrm{kHz})$ 
allow for a rather simple extraction of the amplitude and phase of the signals. In general, this is a cumbersome task in experiments working at optical (or higher) frequencies. Finally, note from Fig. 2 that the relation $J_{\alpha}=2 J_{\beta}$ creates a condition in which any excitation initialized in the edge will tend to stay there for a longer time than when injecting energy in any site of the bulk. This is precisely the result of the topological edge protection [44,45]. In Fig. 2(c), we present the results for an excitation initialized at the edge of the system in the trivial phase, i.e., with the opposite bond dimerization pattern. This simulation verifies that the localization effect is indeed produced by topological protection. Finally, it is important to remark that as in other topologicalinsulator examples, the energy-localization effect shown in Fig. 2(a) becomes stronger as the system's size is increased $[61,62]$.

Coherent transfer of states. A key milestone for the development of scalable quantum computing and communication is the coherent transfer of states among numerous sites in an extended network. Remarkably, it has been shown that if coherence is maintained across many sites, the transfer of quantum states can be obtained with extremely high efficiency [46,63-65]. Indeed, this perfect state transfer can be observed by engineering a qubit chain described by a Hamiltonian of the form $[48,50]$

$$
\begin{aligned}
\hat{H}_{\mathrm{CT}} & =\sum_{n=1}^{N}\left(\varepsilon_{n}-i \Gamma_{n}\right)|n\rangle\left\langle n\left|+\sum_{n=1}^{N} J_{n-1}\right| n-1\right\rangle\langle n| \\
& +\sum_{n=1}^{N} J_{n}|n+1\rangle\langle n|,
\end{aligned}
$$

where the couplings follow the square-root relation: $J_{n}=$ $\frac{\pi}{2 t_{f}} \sqrt{n(N-n)}$, with $t_{f}$ describing the time that an initial onesite excitation takes to be transferred from site $n$ to site $N-$ $n+1$. It is worth mentioning that although the Hamiltonian in Eq. (7) was originally proposed for fermionic qubits [63], its single-excitation nature suggests that it can be implemented in either quantum or classical platforms [42].

In an effort to provide a simple, low-cost platform for simulating quantum state-transfer protocols, we have implemented the Hamiltonian shown in Eq. (7). For this purpose, we have taken $N=7$ oscillators out of the 10 available in the electronic platform. We have arranged them in a chain where all site frequencies, losses, and couplings are characterized by the values presented in the third row of Table I in Appendix A. Note that in order to maintain the same value for all site frequencies, the capacitance in the oscillators takes different values; this is because the corresponding frequencies strongly depend on the couplings, which change with the site positions.

Figure 3 shows the time evolution of an excitation (voltage signal) initialized in the first site of the chain (see the inset in Fig. 3). Note that after $t_{f}=5.6 \mathrm{~s}$, a signal injected into the first site of the chain $(n=1)$ is coherently transferred to the final site $(n=7)$ with an efficiency of 0.61 . This means that $61 \%$ of the total energy is recollected in the intended final site of the lattice. This rather small value is mainly due to the intrinsic losses $(>1 \mathrm{k} \Omega)$ of the general-purpose operational amplifiers (see Appendix B), which could be reduced by making use of low-noise instrumentation amplifiers [66].

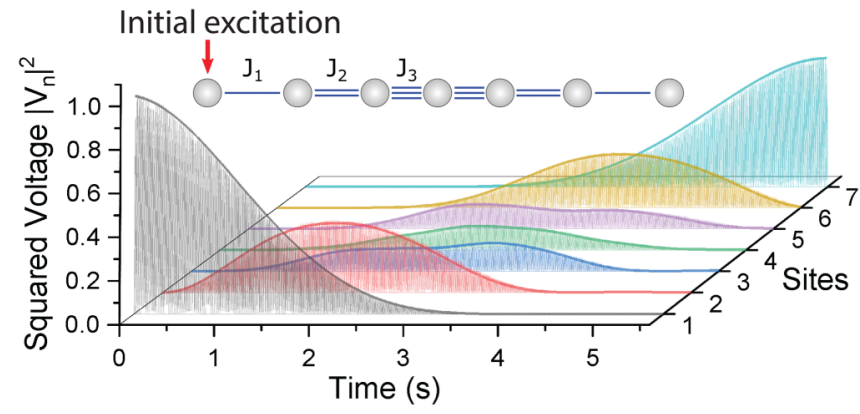

FIG. 3. Time evolution of a voltage signal (excitation) initialized in the first site of the chain $(n=1)$. The inset shows the lattice structure, as well as the initial excitation condition. Note that after the characteristic time $t_{f}=5.6 \mathrm{~s}$, the signal injected into the first site of the chain $(n=1)$ is coherently transferred to the final site $(n=7)$ with an efficiency of 0.61 , that is, $61 \%$ of the total energy is recollected in the intended final site of the lattice.

Photosynthetic energy transport. Finally, we present the simulation of the exciton dynamics in the B800 ring of the purple bacteria LH2 complex. The LH2 complex of Rhodopseudomonas acidophila carries 27 bacteriochlorophyll (BChl) molecules in two concentric rings embedded in the surrounding proteins [52]; 9 of the BChl molecules form the B800 ring (see inset in Fig. 4), which absorbs maximally at $800 \mathrm{~nm}$, and the other 18 molecules form the B850 ring, which absorbs maximally at $850 \mathrm{~nm}$. The BChl molecules in the $\mathrm{B} 850$ ring are closely packed, which leads to strong electronic coupling between adjacent pigments [67], whereas the large distance between adjacent BChl molecules in the B800 ring results in a weak nearest-neighbor coupling.

In the single-excitation basis, the $\mathrm{B} 800$ ring can be described by a tight-binding Hamiltonian of the form [52]

$$
\hat{H}_{\mathrm{B} 800}=\sum_{n=1}^{N}\left(\varepsilon_{n}-i \Gamma_{n}\right)|n\rangle\left\langle n\left|+\sum_{n \neq m}^{N} J_{n m}\right| n\right\rangle\langle m|,
$$

where the excitation energies of the $\mathrm{BChl}$ molecules and the coupling between them are given by $\varepsilon_{n}=12450 \mathrm{~cm}^{-1}$ and

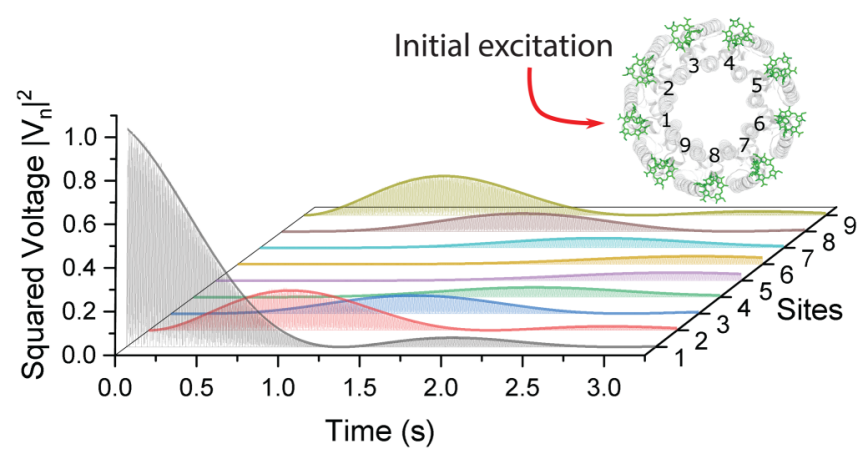

FIG. 4. Time evolution of a voltage signal (excitation) initialized in one of the sites of the B800 ring $(n=1)$. The inset shows the ring structure, as well as the initial excitation condition. Note that the results are presented in a rescaled time window $(3.2 \mathrm{~s})$, which corresponds to a $\sim 0.6$ ps time evolution in the real photosynthetic complex. 


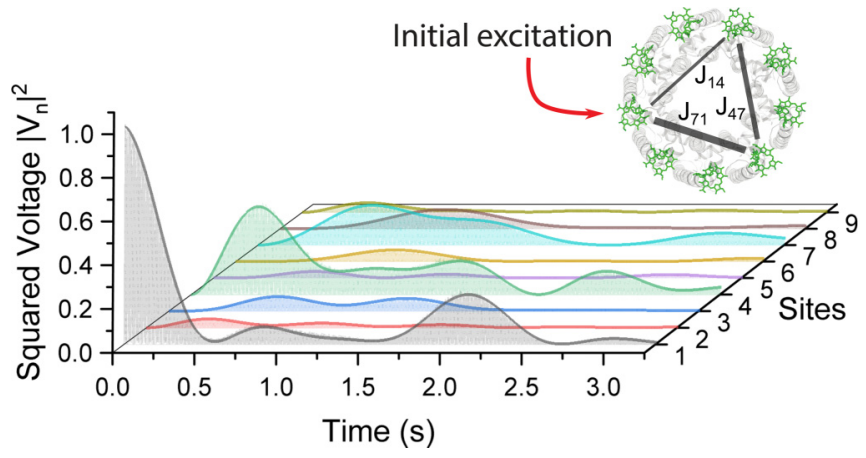

FIG. 5. Time evolution of a voltage signal (excitation) injected into site 1 of the modified B800 complex $(n=1)$. The inset shows the ring structure with artificially introduced long-range interactions between sites 1,4 , and 7 . The values of the couplings are given by the relations $J_{14}=J_{B 800} / 3, J_{47}=2 J_{B 800} / 3$, and $J_{71}=J_{B 800}$.

$J_{n, n-1}=J_{B 800}=-27 \mathrm{~cm}^{-1}$, respectively. To simulate the dynamics described by the Hamiltonian (8), we first note that the rate at which $\mathrm{BChl}$ molecules interact is extremely fast compared to the characteristic frequencies of our platform. Therefore, we introduce a proper rescaling factor, which is found to be $\eta=5.2615 \times 10^{12}$. With this factor, we obtain an excitation energy of $\varepsilon_{n}=446.4 \mathrm{~Hz}$ and a coupling of $J_{B 800}=-0.9 \mathrm{~Hz}$. These parameters are set by making use of the values presented in the fourth row of Table I in Appendix A.

Figure 4 shows the dynamics of a voltage signal (excitation) initialized in one of the sites of the B800 ring, as depicted in the inset. Note that the results are presented in a rescaled time window $(3.2 \mathrm{~s}$ ), which corresponds to a $\sim 0.6 \mathrm{ps}$ time evolution in the real molecular system. Moreover, note that the weak coupling between the BChl molecules in the B800 ring results in a slow propagation of the energy among the sites, thus making the system more susceptible to dissipation effects due to its interaction with an environment [68].

Energy transport beyond nearest-neighbor interactions. We now demonstrate the capability of our platform to simulate single-particle quantum transport in lattices comprising independently addressable long-range interactions. This is demonstrated through a modified Hamiltonian of the B800 complex, comprising artificially introduced couplings among sites 1,4 , and 7 , with their values defined by the relations $J_{14}=J_{B 800} / 3, J_{47}=2 J_{B 800} / 3$, and $J_{71}=J_{B 800}$. Figure 5 shows the dynamics of a voltage signal (excitation) initially injected into site 1 of the modified LH2 complex (shown in the inset of Fig. 5). Note that the presence of the long-range interactions modifies the energy dynamics in such a way that the initial excitation spreads much faster among sites.

Before concluding, we would like to comment on the possibility of extending our electronic platform to larger site numbers and going beyond the single-excitation space. Regarding the former, and given the simplicity of the electronic oscillators and the couplings between them, one could include additional oscillators by directly adding them in the lattice and setting the correct connections. In fact, note that the oscillators and couplings of our platform are structurally the same, as shown in Figs. 6(a) and 6(b) of Appendix A, which allows for a straightforward expansion. Of course, as we continue adding more sites, the corresponding parasitic losses will increase as (a)

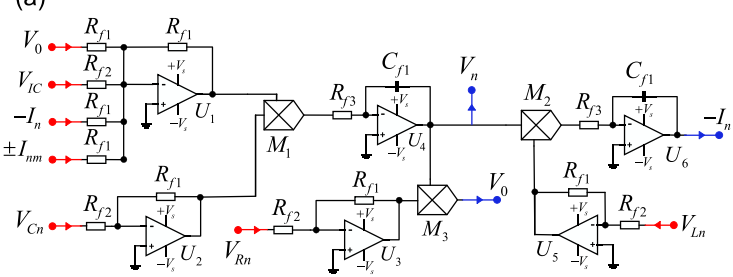

(b)

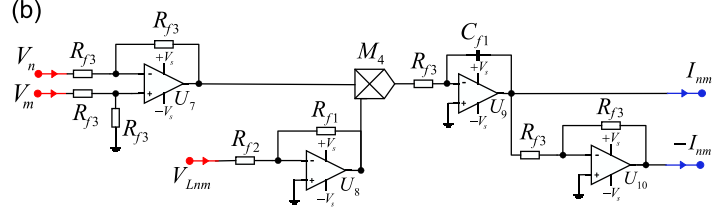

(c)

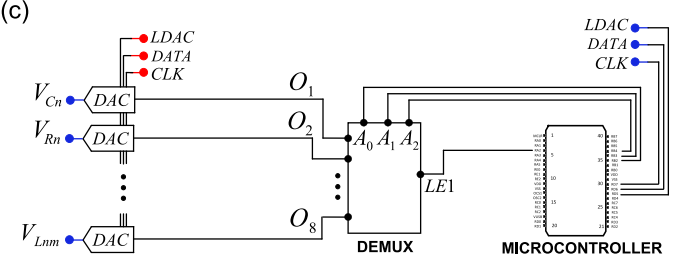

(d)

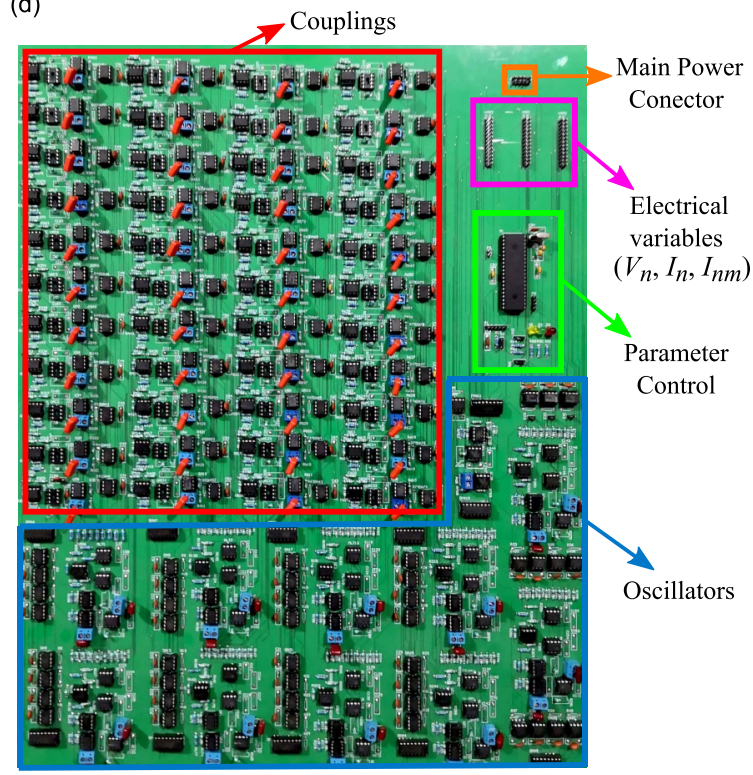

FIG. 6. Schematics for the (a) oscillators, (b) couplings, and (c) parameter digital control. The input signals are indicated with red nodes, whereas the outputs are denoted with blue ones. $R_{f j}, C_{f j}, U_{j}$, and $M_{j}$ stand for resistors, capacitors, operational amplifiers, and analog multipliers, respectively. The voltage in the capacitor $V_{n}$ and the currents across the oscillator inductor $I_{n}$ are the electrical variables of interest, and the label $V_{0}$ refers to the interconnection of feedback signals. $V_{R n}, V_{L n}, V_{C n}$, and $V_{I C}$ are voltage signals generated by the DACs, which allow one to configure the system parameters in an easy and accessible way via software. The communication between the microcontroller and the DACs is performed through the SPI protocol, which makes use of the digital signals LDAC, DATA, and CLK, and the configuration bits (LE $j$, $A_{0}, A_{1}$, and $A_{2}$ ) sent to the demultiplexers. (d) Printed circuit board of 10 fully reconfigurable $R L C$ oscillators. 
well, thus hampering the preservation of the system's coherence. To alleviate this problem, one would need to resort to integrated electronics, which is a mature, well-known technology used for the miniaturization of complex electronic circuits. As for the extension to more than one excitation spaces, a new schematic of the circuit would be needed in order to account for the terms appearing in the equations describing the dynamics of the system. This has been shown, by our group, in a recent experimental implementation of the classical Dicke model [69].

\section{CONCLUSION}

We have presented a versatile, reconfigurable network for the simulation of single-particle quantum transport. By exploiting the negligible decoherence of our network, we have induced complex superpositions and interference effects, thus allowing us to simulate Hamiltonians attributed to important quantum transport dynamics. Because of its robustness and versatility, our device arises as a promising platform for the experimental study of quantum transport phenomena.

\section{ACKNOWLEDGMENTS}

This work was supported by CONACyT under Projects No. CB-2016-01/284372 and No. A1-S-8317, and by DGAPAUNAM under Project UNAM-PAPIIT IN102920. We acknowledge funding from the U.S. Department of Energy, Office of Basic Energy Sciences, Division of Materials Sciences and Engineering under Award No. DE-SC0021069. M.A.Q.-J. and R.J.L.M. would like to thank Gustavo A. Dominguez-Castro for suggesting the implementation of the $\mathrm{SSH}$ model in the electronic platform.

\section{APPENDIX A: CIRCUIT DESIGN AND PARAMETERS}

Our experimental setup comprises a network of 10 inductively coupled $R L C$ oscillators, whose dynamics are governed by Eq. (4) of the main manuscript. The oscillators and couplings are electronically implemented with active networks of operational amplifiers (OPAMPs) and passive linear electrical components. It is worth mentioning that the transfer functions of the basic electrical networks of OPAMPs, namely, adders, integrators, and gains, obey specific mathematical operations. This allows us to interconnect them to build complex sequences of mathematical functions where voltage signals represent physical variables of the system that is being studied. In this representation, the parameters of the system are mapped into passive components within the active networks, such as resistors and capacitors. Consequently, any change in the parameters leads to physically replacing components.

To avoid this, we have merged basic electrical networks with integrated analog multipliers for synthesizing voltage-driven components whose values depend on an external voltage signal provided by digital-to-analog converters (DACs) that communicate with a master microcontroller by the serial peripheral interface (SPI) protocol. Because of this remarkable feature, the initial conditions and the system parameters $R_{n}, L_{n}, C_{n}$, and $L_{n m}$, which control the site frequencies and couplings, can be individually addressed within a wide range of values via software. More importantly, since coupling values can be set to zero, one can control the connection topology between oscillators by enabling or disabling the couplings.

Structurally, our experimental setup is divided in two parts: analog and digital. The former encompasses the oscillators and couplings, both of them built with purely analog electronic components. Figures 6(a) and 6(b) show the general schemes of the electronic circuits for the oscillators and couplings, respectively. There, $R_{f j}, C_{f j}, U_{j}$, and $M_{j}$ stand for metal resistors ( $1 \%$ tolerance), polyester capacitors, generalpurpose operational amplifiers LF353, and analog multipliers AD633JN (four-quadrant voltage multiplier), respectively.

In Fig. 6, the input signals are indicated with red nodes, whereas the outputs are denoted with blue ones. $V_{n}, I_{n}$, and $I_{n m}$ are the electrical variables of interest, namely, the voltage in the capacitor, the currents across the oscillator inductors, and the coupling inductors, respectively. The label $V_{0}$ refers to interconnection of an internal signal. In the experimental setup, the parameters of the oscillators and couplings, $R_{n}$, $L_{n}, C_{n}$, and $L_{n m}$, as well as the initial conditions $V_{n}(0)$, are defined by the values of $R_{f j}, C_{f j}, V_{R n}, V_{L n} V_{C n}$, and $V_{I C}$. These quantities satisfy the following relationships:

$$
\begin{aligned}
\frac{1}{R_{n}} & =\frac{R_{f 1} V_{R n} \phi}{R_{f 2}}, \\
\frac{1}{L_{n}} & =\frac{R_{f 1} V_{L n} \phi}{R_{f 2} R_{f 3} C_{f 1}}, \\
\frac{1}{C_{n}} & =\frac{R_{f 1} V_{C n} \phi}{R_{f 2} R_{f 3} C_{f 1}}, \\
\frac{1}{L_{n m}} & =\frac{R_{f 1} V_{L n m} \phi}{R_{f 2} R_{f 3} C_{f 1}}, \\
V_{n}(0) & =\frac{R_{f 1} V_{I C}}{R_{f 2}},
\end{aligned}
$$

where $\phi=1 / 10$ is a manufacturing default factor of the ana$\log$ multiplier, integrated to avoid saturation of the output voltage. Remarkably, the $R_{f j}$ and $C_{f j}$ devices represent the core configuration of the electronic platform and fix the maximum values that the system parameters can take. Furthermore, the voltage signals $V_{R n}, V_{L n}, V_{C n}$, and $V_{I C}$, coming from the DACs and taking discrete values between 0 and $5 \mathrm{~V}$ with a resolution of $1.22 \mathrm{mV}$, allow one to independently select such parameters from a broad range of possible values within the defined interval. To operate the operational amplifiers and analog multipliers in a convenient bandwidth, the resistor and capacitor values are set to $R_{f 1}=10 \mathrm{k} \Omega, R_{f 2}=5 \mathrm{k} \Omega$, $R_{f 3}=1 \mathrm{k} \Omega$, and $C_{f 1}=0.1 \mu \mathrm{F}$. This configuration allows us to tune the site frequencies from 0 to $1590 \mathrm{~Hz}$. Finally, to energize the device, we make use of a stabilized DC power supply (KEITHLEY triple channel, 2231A-30-3), which feeds the $\pm 12 \mathrm{~V}$ bias voltage $\left(+V_{s},-V_{s}\right)$ to the OPAMPs and the analog multipliers.

As for the digital part, we incorporate digital-to-analog converters (MCP4921, Resolution 12 bits), demultiplexers (SN47HC138N, high-speed CMOS 3-to-8 line decoder), and 
TABLE I. Electrical-component values used in the implementation of the quantum transport protocols presented in the main article.

\begin{tabular}{|c|c|c|}
\hline Experiment & Site frequencies and losses & Coupling coefficients \\
\hline Anderson localization & $\begin{array}{c}C_{n}=1.50 \mathrm{mF} \\
L_{n}=3.35 \mathrm{mH} \\
R_{n}=1 \mathrm{k} \Omega\end{array}$ & $\begin{array}{c}L_{n m}=\left[L_{x}(1-\Delta), L_{x}(1+\Delta)\right] \\
L_{x}=96.05 \mathrm{mH} \\
\Delta=0,0.5,0.9\end{array}$ \\
\hline SSH model & $\begin{array}{c}C_{n}=1.50 \mathrm{mF} \\
L_{n}=3.35 \mathrm{mH} \\
R_{n}=900 \Omega\end{array}$ & $\begin{array}{l}L_{\alpha}=96.05 \mathrm{mH} \\
L_{\beta}=192.1 \mathrm{mH}\end{array}$ \\
\hline Coherent transfer of states & $\begin{array}{c}C_{1}=C_{4}=C_{7}=7.54 \mathrm{mF} \\
C_{2}=C_{3}=C_{5}=C_{6}=7.58 \mathrm{mF} \\
L_{n}=1.11 \mathrm{mH} \\
R_{n}=1.5 \mathrm{k} \Omega\end{array}$ & $\begin{array}{c}L_{12}=L_{67}=321.36 \mathrm{mH} \\
L_{23}=L_{56}=181.97 \mathrm{mH} \\
L_{34}=L_{45}=75.45 \mathrm{mH}\end{array}$ \\
\hline Photosynthetic transport & $\begin{array}{c}C_{n}=1.50 \mathrm{mF} \\
L_{n}=3.35 \mathrm{mH} \\
R_{n}=1 \mathrm{k} \Omega\end{array}$ & $L_{n m}=806.90 \mathrm{mH}$ \\
\hline
\end{tabular}

a microcontroller (PIC18 familiy), which together deal with the parameter and initial condition configurations. Note that in the electronic platform there are 85 configurable parameters, four per each oscillator and 40 possible all-to-all couplings, with each one of them controlled by voltage signals coming from the DAC $\left(V_{R n}, V_{L n}, V_{C n}\right.$, and $\left.V_{I C}\right)$. To satisfy this demand, the enable/disable terminal of each DAC is connected to a digital bus managed by demultiplexers; in this way, with only 16 lines of the microcontroller, we can select a particular DAC, setting the properly configuration bits $L E j, A_{0}, A_{1}$, and $A_{2}$ to the demultiplexers, as well as to transmit a desired output voltage to the DAC through the SPI protocol using the control and data bits, namely, LDAC, DATA, and CLK [see Fig. 6(c)].

To ensure a strong connection among the electronic components, we design and manufacture a printed circuit board $(\mathrm{PCB}, 40 \times 50 \mathrm{~cm})$ where electronic devices are mounted and soldered. The PCB was designed using ALTIUM software and fabricated with a computer numerical control (CNC) laser. The electronic realization of the 10 fully reconfigurable $R L C$ oscillators on the PCB is shown in Fig. 6(d). The module controlling the parameters and initial conditions is indicated with a green square, whereas the analog oscillators and couplings are signaled with blue and red, respectively. Both the analog and digital modules of our experimental setup are energized through the main power connector (orange squared). The acquisition of the electrical variables (magenta square) is performed with a Digilent oscilloscope (Analog Discovery 2 ), which directly transfers the information to a computer by USB connection.

To conclude this section, we provide (in Table I) detailed information regarding the electronic-component values needed for the implementation of the experiments described in the main text.

\section{APPENDIX B: ENERGY-LOSS ESTIMATION}

In this section, we provide a thorough description of how the unavoidable losses, present in our electronic platform, can be accounted for in the quantum tight-binding network model. Let us consider the energy contained in the whole circuit, which is given by

$$
Q_{\mathrm{cl}}(t)=\frac{1}{2} \sum_{m \neq n} C_{n} V_{n}^{2}+L_{n} I_{n}^{2}+L_{n m} I_{n m}^{2} .
$$

As one might expect, in the presence of losses (or resistance), the total energy of the system will decay following an exponential behavior [57]. Of course, in the absence of resistance, the total energy is conserved. Remarkably, in the quantum model, a quantity that follows the same behavior in the presence (or absence) of losses is the trace of the density matrix. We define an energylike measure of the quantum system, by making use of the expression

$$
Q_{\mathrm{q}}(t)=\sum_{n}\left|c_{n}\right|^{2}
$$

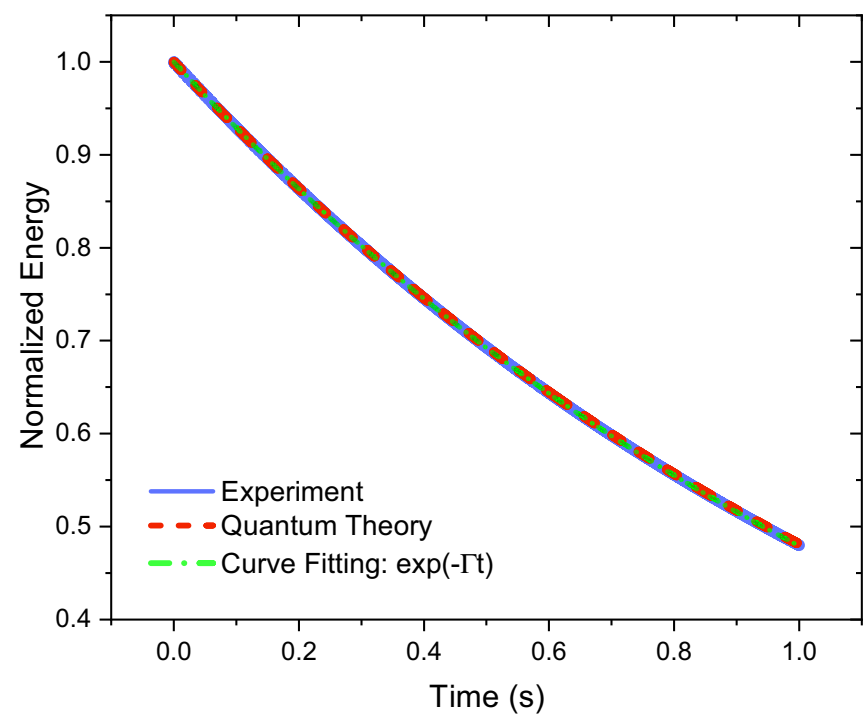

FIG. 7. Time evolution of the normalized energy in the electronic platform (solid line) and the quantum dissipative model (dashed line). The dash-dotted line shows an exponential curve fitting. The parameters used for obtaining the energy curves are those used in the experimental implementation of the SSH model. 
It is well known that for a closed quantum system, the trace of the system's density matrix is conserved, whereas for a system affected by a dissipative environment, the trace of the reduced density matrix (defined as the density matrix obtained after the environment's degrees of freedom are traced out) decays exponentially [70]. Along this line, the simplest way of introducing a dissipative process in a quantum system is by including a non-Hermitian term, in the closed system's Hamiltonian, of the form

$$
\hat{H}_{\text {loss }}=-\frac{i}{2} \sum_{n} \Gamma_{n}|n\rangle\langle n|,
$$

with $\Gamma_{n}$ describing the rate at which energy is dissipated to the system's environment.

Figure 7 shows the time evolution of the normalized energy for the electrical circuit (blue solid line) and the trace of the open system's density matrix (red dashed line). Note that both curves follow the same exponentially decaying behavior (described by the green dotted curve fitting), which allows us to establish the relation $\Gamma=1 /(R C)$, where $R=$ $R_{n}$ and $C=C_{n}$ stand for the resistance and capacitance of each electrical oscillator in the device, respectively. This important result is what allows us to include the effects of the electronic parasitic losses into the quantum tight-binding model.
[1] P. Hänggi and F. Marchesoni, Artificial Brownian motors: Controlling transport on the nanoscale, Rev. Mod. Phys. 81, 387 (2009).

[2] N. Lambert, Y. N. Chen, Y. C. Cheng, C. M. Li, G. Y. Chen, and F. Nori, Quantum biology, Nat. Phys. 9, 10 (2012).

[3] J. Cao et al., Quantum biology revisited, Sci. Adv. 6, eaaz4888 (2020).

[4] A. F. Collings and C. Critchley, Artificial Photosynthesis: From Basic Biology to Industrial Application (WILEY-VCH, Weinheim, 2005).

[5] H. Hodaei, A. U. Hassan, S. Wittek, H. Garcia-Gracia, R. El-Ganainy, D. N. Christodoulides, and M. Khajavikhan, Enhanced sensitivity at higher-order exceptional points, Nature (London) 548, 187 (2017).

[6] S. Pirandola, B. R. Bardhan, T. Gehring, C. Weedbrook, and S. Lloyd, Advances in photonic quantum sensing, Nat. Photon. 12, 724 (2018).

[7] C. Chen and J. Wang, Optical biosensors: An exhaustive and comprehensive review, Analyst 145, 1605 (2020).

[8] A. Aspuru-Guzik and P. Walther, Photonic quantum simulators, Nat. Phys. 8, 285 (2012).

[9] C. H. Lee, S. Imhof, C. Berger, F. Bayer, J. Brehm, L. W. Molenkamp, T. Kiessling, and R. Thomale, Topolectrical circuits, Commun. Phys. 1, 39 (2018).

[10] J. Wang, F. Sciarrino, A. Laing, and M. G. Thompson, Integrated photonic quantum technologies, Nat. Photon. 14, 273 (2020).

[11] A. W. Elshaari, W. Pernice, K. Srinivasan, O. Benson, and V. Zwiller, Hybrid integrated quantum photonic circuits, Nat. Photon. 14, 285 (2020).

[12] F. Caruso, N. Spagnolo, C. Vitelli, F. Sciarrino, and M. B. Plenio, Simulation of noise-assisted transport via optical cavity networks, Phys. Rev. A 83, 013811 (2011).

[13] S. Viciani, M. Lima, M. Bellini, and F. Caruso, Observation of Noise-Assisted Transport in an All-Optical Cavity-Based Network, Phys. Rev. Lett. 115, 083601 (2015).

[14] F. Beaudoin, A. Blais, and W. A. Coish, Hamiltonian engineering for robust quantum state transfer and qubit readout in cavity QED, New J. Phys. 19, 023041 (2017).

[15] F. Zahringer, G. Kirchmair, R. Gerritsma, E. Solano, R. Blatt, and C. F. Roos, Realization of a Quantum Walk with One and Two Trapped Ions, Phys. Rev. Lett. 104, 100503 (2010).
[16] R. Blatt and C. F. Roos, Quantum simulations with trapped ions, Nat. Phys. 8, 277 (2012).

[17] N. Trautmann and P. Hauke, Trapped-ion quantum simulation of excitation transport: Disordered, noisy, and long-range connected quantum networks, Phys. Rev. A 97, 023606 (2018).

[18] C. H. Alderete, S. Singh, N. H. Nguyen, D. Zhu, R. Balu, C. Monroe, C. M. Chandrashekar, and N. M. Linke, Quantum walks and Dirac cellular automata on a programmable trappedion quantum computer, Nat. Commun. 11, 3720 (2020).

[19] M. Lewenstein, A. Sanpera, V. Ahufinger, B. Damski, A. Sen, and U. Sen, Ultracold atomic gases in optical lattices: Mimicking condensed matter physics and beyond, Adv. Phys. 56, 243 (2007).

[20] I. Bloch, J. Salibard, and S. Nascimbene, Quantum simulations with ultracold quantum gases, Nat. Phys. 8, 267 (2012).

[21] P. M. Preiss, R. Ma, M. E. Tai, A. Lukin, M. Rispoli, P. Zupancic, Y. Lahini, R. Islam, and M. Greiner, Strongly correlated quantum walks in optical lattices, Science 347, 1229 (2015).

[22] S. Dadras, A. Gresch, C. Groiseau, S. Wimberger, and G. S. Summy, Quantum Walk in Momentum Space with a BoseEinstein Condensate, Phys. Rev. Lett. 121, 070402 (2018).

[23] B. Peropadre, G. G. Guerreschi, J. Huh, and A. Aspuru-Guzik, Proposal for Microwave Boson Sampling, Phys. Rev. Lett. 117, 140505 (2016).

[24] Z. Yan et al., Strongly correlated quantum walks with a 12-qubit superconducting processor, Science 364, 753 (2019).

[25] M. Kjaergaard, M. E. Schwartz, J. Braumüller, P. Krantz, J. I.J. Wang, S. Gustavsson, and W. D. Oliver, Superconducting qubits: Current state of play, Annu. Rev. Condens. Matter Phys. 11, 369 (2020).

[26] G. Volpe and G. Volpe, Simulation of a Brownian particle in an optical trap, Am. J. Phys. 81, 224 (2013).

[27] P. H. Jones, O. M. Marago, and G. Volpe, Optical Tweezers. Principles and Applications (Cambridge University Press, Padstow Cornwall, 2015).

[28] R. de J. León-Montiel and P. A. Quinto-Su, Noise-enabled optical ratchets, Sci. Rep. 7, 44287 (2017).

[29] A. V. Arzola, M. Villasante-Barahona, K. Volke-Sepulveda, P. Jakl, and P. Zemanek, Omnidirectional Transport in Fully Reconfigurable Two Dimensional Optical Ratchets, Phys. Rev. Lett. 118, 138002 (2017). 
[30] M. G. Sánchez-Sánchez, R. de J. León-Montiel, and P. A. Quinto-Su, Phase Dependent Vectorial Current Control in Symmetric Noisy Optical Ratchets, Phys. Rev. Lett. 123, 170601 (2019).

[31] T. Schwartz, G. Bartal, S. Fishman, and M. Segev, Transport and Anderson localization in disordered two-dimensional photonic lattices, Nature (London) 446, 52 (2007).

[32] A. Peruzzo, M. Lobino, J. C. F. Matthews, N. Matsuda, A. Politi, K. Pulios, X.-Q. Zhou, Y. Lahini, N. Ismail, K. Worhoff, Y. Bromberg, Y. Silberberg, M. G. Thompson, and J. L. O'Brien, Quantum walks of correlated photons, Science 329, 1500 (2010).

[33] A. Schreiber, K. N. Cassemiro, V. Potocek, A. Gabris, I. Jex, and C. Silberhorn, Decoherence and Disorder in Quantum Walks: From Ballistic Spread to Localization, Phys. Rev. Lett. 106, 180403 (2011)

[34] L. Sansoni, F. Sciarrino, G. Vallone, P. Mataloni, A. Crespi, R. Ramponi, and R. Osellame, Two-Particle Bosonic-Fermionic Quantum Walk Via Integrated Photonics, Phys. Rev. Lett. 108, 010502 (2012).

[35] A. Crespi, R. Osellame, R. Ramponi, V. Giovannetti, R. Fazio, L. Sansoni, F. D. Nicola, F. Sciarrino, and P. Mataloni, Anderson localization of entangled photons in an integrated quantum walk, Nat. Photon. 7, 322 (2013).

[36] M. C. Rechtsman, J. M. Zeuner, Y. Plotnik, Y. Lumer, D. Podolsky, F. Dreisow, S. Nolte, M. Segev, and A. Szameit, Photonic Floquet topological insulators, Nature (London) 496, 196 (2013).

[37] D. N. Biggerstaff, R. Heilmann, A. A. Zecevik, M. Grafe, M. A. Broome, A. Fedrizzi, S. Nolte, A. Szameit, A. G. White, and I. Kassal, Enhancing coherent transport in a photonics network using controllable decoherence, Nat. Commun. 7, 11282 (2016).

[38] F. Caruso, A. Crespi, A. G. Ciriolo, F. Sciarrino, and R. Osellame, Fast escape of a quantum walker from an integrated photonic maze, Nat. Commun. 7, 11682 (2016).

[39] A. Perez-Leija, D. Guzman-Silva, R. de J. León-Montiel, M. Grafe, M. Heinrich, H. Moya-Cessa, K. Busch, and A. Szameit, Endurance of quantum coherence due to particle indistinguishability in noisy quantum networks, npj Quantum Inf. 4, 45 (2018).

[40] M. A. Quiroz-Juárez, A. Perez-Leija, K. Tschernig, B. M. Rodríguez-Lara, O. S. Magaña-Loaiza, K. Busch, Y. N. Joglekar, and R. de J. León-Montiel, Exceptional points of any order in a single, lossy waveguide beam splitter by photonnumber-resolved detection, Photon. Res. 7, 862 (2019).

[41] N. C. Harris, G. R. Steinbrecher, M. Prabhu, Y. Lahini, J. Mower, D. Bunandar, C. Chen, F. N. C. Wong, T. Baehr-Jones, M. Hochberg, S. Lloyd, and D. Englund, Quantum transport simulations in a programmable nanophotonic processor, Nat. Photon. 11, 447 (2017).

[42] R. de J. León-Montiel, A. Vallés, H. M. Moya-Cessa, and J. P. Torres, Coherent delocalization: Views of entanglement in different scenarios, Laser Phys. Lett. 12, 085204 (2015).

[43] P. W. Anderson, Absence of diffusion in certain random lattices, Phys. Rev. 109, 1492 (1958).

[44] W. P. Su, J. R. Schrieffer, and A. J. Heeger, Solitons in Polyacetylene, Phys. Rev. Lett. 42, 1698 (1979).

[45] W. P. Su, J. R. Schrieffer, and A. J. Heeger, Soliton excitations in polyacetylene, Phys. Rev. B 22, 2099 (1980).
[46] M. A. Nielsen and I. L. Chuang, Quantum Computation and Quantum Information (Cambridge University Press, New York, 2000).

[47] L.-M. Liang and C.-Z. Li, Realization of quantum swap gate between flying and stationary qubits, Phys. Rev. A 72, 024303 (2005).

[48] M. Christandl, N. Datta, A. Ekert, and A. J. Landahl, Perfect State Transfer in Quantum Spin Networks, Phys. Rev. Lett. 92, 187902 (2004).

[49] M. B. Plenio, J. Hartley, and J. Eisert, Dynamics and manipulation of entanglement in coupled harmonic systems with many degrees of freedom, New J. Phys. 6, 36 (2004).

[50] A. Perez-Leija, R. Keil, A. Kay, H. Moya-Cessa, S. Nolte, L.-C. Kwek, B. M. Rodriguez-Lara, A. Szameit, and D. N. Christodoulides, Coherent quantum transport in photonic lattices, Phys. Rev. A 87, 012309 (2013).

[51] T. Tian, S. Lin, L. Zhang, P. Yin, P. Huang, C. Duan, L. Jiang, and J. Du, Perfect coherent transfer in an on-chip reconfigurable nanoelectromechanical network, Phys. Rev. B 101, 174303 (2020).

[52] Y. C. Cheng and R. J. Silbey, Coherence in the b800 Ring of Purple Bacteria lh2, Phys. Rev. Lett. 96, 028103 (2006).

[53] J. S. Briggs and A. Eisfeld, Equivalence of quantum and classical coherence in the electronic energy transfer, Phys. Rev. E 83, 051911 (2011).

[54] R. de J. León-Montiel and J. P. Torres, Highly Efficient NoiseAssisted Energy Transport in Classical Oscillator Systems, Phys. Rev. Lett. 110, 218101 (2013).

[55] R. de J. León-Montiel, M. A. Quiroz-Juárez, R. QuinteroTorres, J. L. Domínguez-Juárez, H. M. Moya-Cessa, J. P. Torres, and J. L. Aragón, Noise-assisted energy transport in electrical oscillator networks with off-diagonal dynamical disorder, Sci. Rep. 5, 17339 (2015).

[56] M. Quiroz-Juárez, J. Aragón, R. de J. León-Montiel, R. Vazquez-Medina, J. Domínguez-Juárez, and R. Quintero-Torres, Emergence of a negative resistance in noisy coupled linear oscillators, Europhys. Lett. 116, 50004 (2017).

[57] R. de J. León-Montiel, M. A. Quiroz-Juárez, J. L. DomínguezJuárez, R. Quintero-Torres, J. L. Aragón, A. K. Harter, and Y. N. Joglekar, Observation of slowly decaying eigenmodes without exceptional points in Floquet dissipative synthetic circuits, Commun. Phys. 1, 88 (2018).

[58] L. Martin, G. D. Giuseppe, A. Perez-Leija, R. Keil, F. Dreisow, M. Heinrich, S. Nolte, A. Szameit, A. F. Abouraddy, D. N. Christodoulides, and B. E. A. Saleh, Anderson localization in optical waveguide arrays with off-diagonal coupling disorder, Opt. Express 19, 13636 (2011).

[59] J. K. Asboth, L. Oroszlany, and A. Palyi, The Su-SchriefferHeeger (SSH) model, A Short Course on Topological Insulators, Lecture Notes in Physics Vol. 919 (Springer, Cham, 2016).

[60] S. Liu, W. Gao, Q. Zhang, S. Ma, L. Zhang, C. Liu, Y. J. Xiang, T. J. Cui, and S. Zhang, Topologically protected edge state in two-dimensional Su-Schrieffer-Heeger circuit, Research 2019, 8609875 (2019).

[61] H. Luo, Advanced Topological Insulators (John Wiley \& Sons, Inc., Hoboken, NJ, 2019).

[62] P. Reséndiz-Vázquez, K. Tschernig, A. Perez-Leija, K. Busch, and R. de J. León-Montiel, Topological protection in 
non-Hermitian Haldane honeycomb lattices, Phys. Rev. Res. 2, 013387 (2020).

[63] S. Bose, Quantum Communication through an Unmodulated Spin Chain, Phys. Rev. Lett. 91, 207901 (2003).

[64] S. Bose, Quantum communication through spin chain dynamics: An introductory overview, Contemp. Phys. 48, 13 (2007).

[65] A. Kay, Perfect, efficient, state transfer and its application as a constructive tool, Intl. J. Quantum Inf. 8, 641 (2010).

[66] R. F. Coughlin and F. F. Driscoll, Operational Amplifiers and Linear Integrated Circuits, 6th ed. (Prentice Hall, New Jersey, 2001).
[67] G. D. Scholes, I. R. Gould, R. J. Cogdell, and G. R. Fleming, Ab initio molecular orbital calculations of electronic couplings in the LH2 bacterial light-harvesting complex of Rps. acidophila, J. Phys. Chem. B 103, 2543 (1999).

[68] R. Croce, R. van Grondelle, H. van Amerongen, and I. van Stokkum, Light Harvesting in Photosynthesis (CRC, Boca Raton, FL, 2018).

[69] M. A. Quiroz-Juárez, J. Chávez-Carlos, J. L. Aragón, J. G. Hirsch, and R. de J. León-Montiel, Experimental realization of the classical Dicke model, Phys. Rev. Res. 2, 033169 (2020).

[70] H.-P. Breuer and F. Petruccione, The Theory of Open Quantum Systems (Oxford University Press, Oxford, 2002). 